\title{
ESSENTIALLY HERMITIAN MATRICES AND INCLUSION RELATIONS OF $C$-NUMERICAL RANGES
}

\author{
WAI-SHUN CHEUNG
}

Abstract. Let $\mathbf{M}$ denote the set of all $n \times n$ complex matrices and $\mathbf{M}_{n}^{0}$ denote the set of $n \times n$ matrices with trace 0 . For any $C \in \mathbf{M}_{n}^{0}$, there exists a maximal $v(C) \geqslant 0$ such that

$$
v(C) W_{D}(A) \subseteq\|D\|_{F} W_{C}(A)
$$

whenever $D \in \mathbf{M}_{n}^{0}$ and $A \in \mathbf{M}_{n}$. Here $W_{C}(A)$ denotes the $C$-numerical range of $A$ and $\|D\|_{F}$ denotes the Frobenius norm of $D$. Moreover $v(C)=0$ if and only if $C$ is essentially hermitian.

To prove the above result, we have obtained a new characterisation of essentially hermitian matrices.

Mathematics subject classification (2000): 15A57, 47A12.

Keywords and phrases: essentially hermitian matrix, numerical range.

\section{REFERENCES}

[1] Y. H. Au-Yeung And N. K. Tsing, A conjecture of Marcus on the generalized numerical range, Linear and Multilinear Algebra, 14 (1983), 235-239.

[2] W. S. Cheung, Some Geometrical Aspects Of and Inclusion Relations For Generalized Numerical Ranges, M. Phil Thesis, The University of Hong Kong, 1996.

[3] W. S. Cheung AND N. K. Tsing, The $C$-numerical range of matrices is star-shaped, Linear and Multilinear Algebra, 41, 3 (1996), 245-250.

[4] M. GOLDBERG AND E. G. STRAUS, Elementary inclusion relations for generalized numerical ranges, Lin. Alg. Appl., 18 (1977), 1-18.

[5] R. Horn And C. R. Johnson, Topics in Matrix Analysis, Cambridge University Press, Cambridge, 1991.

[6] C.-K. LI, C-numerical ranges and C-numerical radii, Linear and Multilinear Algebra, 37 (1994), $51-82$.

[7] C.-K. Li AND N. K. Tsing, Matrices with circular symmetry on their unitary similarity orbits and C-numerical ranges, Proc. Amer. Math. Soc., 111 (1991), 19-28.

[8] Y. T. Poon, Another proof of a result of Westwick, Linear and Multilinear Algebra, 9 (1980), 35-37.

[9] N. K. Tsing, The constrained bilinear form and the C-numerical range, Linear Algebra and Its Applications, 56 (1984), 195-206.

[10] R. Westwick, A theorem on numerical ranges, Linear and Multilinear Algebra, 2 (1975), 311-315. 